\title{
Terapia celular en la enfermedad de Parkinson y los factores que influyen en su éxito
}

Tamara Jorquiera - Johnson ${ }^{1}$

\section{RESUMEN}

Objetivo: Determinar los factores que influyen en la mejoría de pacientes con enfermedad de Parkinson tratados con células madre adultas autólogas de médula ósea por infusión intra arterial.

Material y Métodos: Estudio observacional, analítico y retrospectivo. Se evaluaron los 58 pacientes con enfermedad de Parkinson que recibieron terapia celular en el Instituto de Radiología Intervencionista Brazzini, entre julio de 2006 y marzo de 2010. La mejoría se determinó comparando los valores UPDRS previos al procedimiento y los mejores valores del seguimiento del paciente. Se usó el SPSS versión 15.0 para el análisis de relación entre la mejoría y los factores intrínsecos del paciente y su enfermedad. $\mathrm{P}<0.05$ para determinar significancia estadística.

Resultados: El 62\% de los pacientes obtuvo una buena mejoría de los valores de UPDRS (más de 50\% de mejoría). No se encontraron diferencias significativas $(p>0.05)$ en los resultados de mejoría según sexo. Se encontraron diferencias significativas $(p<0.05)$, en la mejoría según grupos etáreos. Los pacientes con más mejoría fueron los del grupo de 50 a 59 años y los de poca mejoría fueron los del grupo de 70 a 79 años. Los factores intrínsecos como diskinesia grave, hipertensión arterial, diabetes mellitus, cáncer en remisión, antecedentes familiares de EP, mes de seguimiento del resultado no influyeron en el resultado de mejoría ( $p>0.05)$.

Conclusión: Los factores que influyen en la mejoría de los pacientes tratados con terapia celular para EP son la edad de los pacientes al inicio de la enfermedad y la edad al tratamiento. Se recomienda usar estos factores para elegir a los pacientes que recibirán esta terapia. (Horiz Med 2015; 15(4): 44-51)

Palabras clave: Enfermedad de Parkinson, Terapia celular. (Fuente: DeCS BIREME).

Cell therapy in Parkinson's disease and factors affecting the success

\section{ABSTRACT}

Objective: To determine the factors that influence improvement of patients with Parkinson's disease treated with autologous adult stem cells from bone marrow by intra-arterial infusion.

Material and Methods: An observational, analytical and retrospective study. We evaluated 58 patients with Parkinson's disease (PD) who received cell therapy at the Brazzini Institute of Interventional Radiology, between July 2006 and March 2010. The improvement was determined by comparing the UPDRS values before the procedure and the best values of patient monitoring. SPSS version 15.0 was used for analysis of relationship between improvement and intrinsic factors of the patient and his disease. $\mathrm{P}<0.05$ to determine statistical significance.

Results: $62 \%$ of patients achieved a good improvement in UPDRS values (more than $50 \%$ improvement). No significant differences $(p>0.05)$ in the results of improvement were found by gender. Significant differences $(p<0.05)$ in the improvement according to age groups. Patients with more improvement were in the group of 50-59 years and those with little improvement were in the group of 70-79 years. Intrinsic factors such as severe dyskinesia, hypertension, diabetes mellitus, cancer in remission, family history of PD and month of follow-up did not influence the result of improvement ( $p>0.05)$.

Conclusion: Factors influencing the improvement in patients treated with cell therapy for PD are patient's age at disease onset and age at treatment. We recommend using these factors to choose patients who receive this therapy. (Horiz Med 2015; 15(4): 44-51)

Key words: Parkinson's disease, cell therapy. (Source: MeSH NLM).

1 Médico Cirujano, Departamento de Investigación del Instituto Brazzini Radiólogos, Docente FMH USMP 


\section{INTRODUCCIÓN}

La enfermedad de Parkinson (EP) afecta hasta el $2 \%$ de la población mayor de 65 años, con una mayor incidencia en hombres que en mujeres, pero de similar distribución en todos los grupos étnicos (1-3).

Es un cuadro neurodegenerativo crónico que se caracteriza por la interrupción de la transmisión de dopamina en los ganglios basales debido a la afección de la sustancia nigra, lo que reduce la cantidad de neuronas dopaminérgicas (1-6).

La sustancia nigra es el lugar del cerebro que controla el movimiento, por lo tanto los síntomas son temblor, rigidez, aquinesia, inestabilidad postural, pérdida del balance y de la coordinación (1-3).

Actualmente, el tratamiento se basa en el reemplazo de la sustancia faltante (dopamina) con un fármaco precursor de ella (levodopa). Esto no disminuye la velocidad de pérdida neuronal aunque presenta efectos secundarios, sobre todo a largo plazo, y los pacientes están destinados a tomar la medicación de por vida, lo que hace que estos efectos sean muy frecuentes $(2,7)$.

Por otro lado, las células madre o progenitoras son aquellas células que se caracterizan por ser poco diferenciadas e inmaduras y poseer una capacidad de división manteniendo un estado indiferenciado (8).

Existen tres tipos de células madre según su capacidad de diferenciación (9): Totipotenciales y Pluripotenciales, que son células embrionarias y las células Mutipotenciales, 0 adultas.

Enciertas condiciones, fisiológicas o experimentales, pueden transformarse en células diferenciadas gracias a un proceso celular específico llamado diferenciación (10).

Se ha demostrado que el cerebro cuenta con sus propias células madre en las regiones subventricular y del hipocampo. Estas son las responsables de la regeneración del tejido de manera fisiológica, por lo que se ha planteado la posibilidad que las células madre extraneurales tuvieran también un efecto en la regeneración neuronal (11-13).

El trasplante celular de diversas fuentes (embrionarias o adultas) ha sido propuesto como terapia para los daños producidos en el sistema nervioso central (SNC), con resultados prometedores en ensayos pre-clínicos y clínicos (14).

Entre estos trasplantes, los autólogos no tienen los problemas éticos del uso de células embrionarias ni los problemas de compatibilidad.

La infusión de células madre autólogas de la médula ósea ha ofrecido resultados alentadores (15-16) y cuenta con el potencial para ayudar a la regeneración de los tejidos mediante un procedimiento mínimamente invasivo y con pocas complicaciones.

Sin embargo, no ha sido posible todavía, pronosticar el éxito que tendrían estos tratamientos con células madre autólogas. Se debe buscar cuales son las características clínicas y demográficas de los pacientes tratados y relacionarlos con su mejoría.

Por lo tanto, en este estudio analítico se identificaron los factores intrínsecos que influyen en la mejoría de pacientes con enfermedad de Parkinson tratados con células madre adultas autólogas provenientes de la médula ósea. Buscando conocer cuáles son los pacientes que se beneficiarían más con este tipo de tratamiento.

\section{MATERIAL Y MÉTODOS}

Estudio observacional, analítico y retrospectivo. Se evaluaron los 58 pacientes con enfermedad de Parkinson que recibieron terapia celular en el Instituto de Radiología Intervencionista Brazzini, entre julio de 2006 y marzo de 2010, que cumplieron con los criterios de inclusión y exclusión que se encuentran en la tabla 1. 
Tabla 1. Criterios de inclusión y exclusión.

\section{Criterios de inclusión}

- Pacientes de ambos sexos.

- Pacientes mayores de 18 años.

- Pacientes con buen estado general según evaluaciones médicas, análisis de laboratorio y estudios de imagen. La comorbilidad será consultada con un especialista para descartar complicaciones.

- Datos clínicos y patológicos completos.

\section{Criterios de exclusión}

- Historia clínica y seguimiento incompleto del paciente.

- Diagnóstico y tratamiento fuera del período de estudio

- Pacientes con: depleción de la médula ósea o discrasias sanguíneas, insuficiencia cardiáca, respiratoria, hepática o renal, cáncer o enfermedades infecciosas.

- Adulto mayor con alto grado de fragilidad.

El Instituto Brazzini usó el score de la UPDRS, por las siglas en inglés de la Escala de Evaluación Unificada para la Enfermedad de Parkinson (Unified Parkinson Disease Rating Scale), para medir la mejoría del paciente luego de la terapia celular.

Este score permite seguir el curso longitudinal de la enfermedad del Parkinson y evalúa cuatro áreas: i) estado mental, conducta y humor, ii) actividades de la vida diaria, iii) exploración de aspectos motores y iv) complicaciones de la terapia farmacológica como disquinesias y fluctuaciones clínicas, con puntajes que van desde 0 (ninguna incapacidad) hasta 199 (incapacidad total).

El seguimiento se había realizado durante las semanas uno y dos, así como en los meses uno, tres, seis y doce después de la terapia celular. La mejoría se determinó comparando los valores UPDRS previos al procedimiento y los mejores valores del seguimiento de cada paciente.

Para este estudio, se elaboraron tablas de frecuencia y proporciones (porcentajes) para las variables cualitativas. El análisis estadístico empleó el programa Statistical Package for Social Sciences (SPSS) versión 15.0. Se realizó el análisis de relación bivariada con el coeficiente de correlación de Pearson y la prueba de Chi-cuadrado para evaluar la asociación entre grupos formados al categorizar las variables continuas. La prueba de Wilcoxon se utilizó para verificar si existe diferencia entre el score UPDRS medido antes y después del tratamiento.

Finalmente se realizó un análisis de relación multivariada empleando regresión logística múltiple para comprobar la existencia de relaciones entre las variables de interés. El nivel de significancia estadística fue al 95\%, $p$-valor $<0.05$. El estudio fue evaluado por el comité interno de ética del Instituto Brazzini, asegurándose además la confidencialidad de la información de los pacientes.

\section{RESULTADOS}

El mayor porcentaje de los pacientes fueron varones (69\%) y más del $50 \%$ de pacientes tenían entre 50 y 69 años. Menos del $5 \%$ de pacientes tenían menos de 39 años o más de 80 años, como se puede ver en la tabla 2.

Tabla 2. Pacientes con enfermedad de Parkinson según edades

\begin{tabular}{ccc} 
Edades & $\mathbf{n}$ & $\%$ \\
\hline $30-39$ & 3 & 5,2 \\
$40-49$ & 4 & 6,9 \\
$50-59$ & 17 & 29,3 \\
$60-69$ & 21 & 36,2 \\
$70-79$ & 12 & 20,7 \\
$80-89$ & 1 & 1,7 \\
\hline Total & 58 & 100,0
\end{tabular}

Como se puede observar en la tabla 3, al momento de la terapia celular el promedio de edad fue 61.41 años, con una desviación estándar de 10.54 años, el promedio de edad, al inicio de la enfermedad, fue 52.14 años, con un desviación estándar de 11.31 años; el tiempo promedio de enfermedad al 
implante fue 9.28, años con una desviación estándar de 5.22 años, el mes de seguimiento promedio, en su mejor mes, fue 4.12 meses, con una desviación estándar de 3.42 meses y, por último, el porcentaje promedio de mejoría fue $59 \%$, con una desviación estándar de $25 \%$.

Tabla 3. Datos descriptivos de los pacientes con enfermedad de Parkinson

\begin{tabular}{|c|c|c|c|c|c|}
\hline Variable & $n$ & Minimo & Máximo & Media & DS \\
\hline Edad al implante & 58 & 38 & 81 & 61.41 & 10.54 \\
\hline $\begin{array}{l}\text { Edad al inicio de } \\
\text { la enfermedad }\end{array}$ & 58 & 26 & 75 & 52.14 & 11.31 \\
\hline $\begin{array}{l}\text { Tiempo de enfermedad } \\
\text { al implante }\end{array}$ & 58 & 1 & 25 & 9.28 & 5.22 \\
\hline $\begin{array}{l}\text { Mes de seguimiento } \\
\text { en su mejor mes }\end{array}$ & 58 & 1 & 18 & 4.12 & 3.42 \\
\hline Porcentaje de mejoría & 58 & 0.05 & 0.99 & 0.59 & 0.25 \\
\hline
\end{tabular}

Se consideró como un buen resultado de la terapia celular, cuando los pacientes lograron $50 \%$ o más de mejoría en el score UPDRS. La mayoría de pacientes (62\%), tuvieron un buen resultado, ver la figura 1.

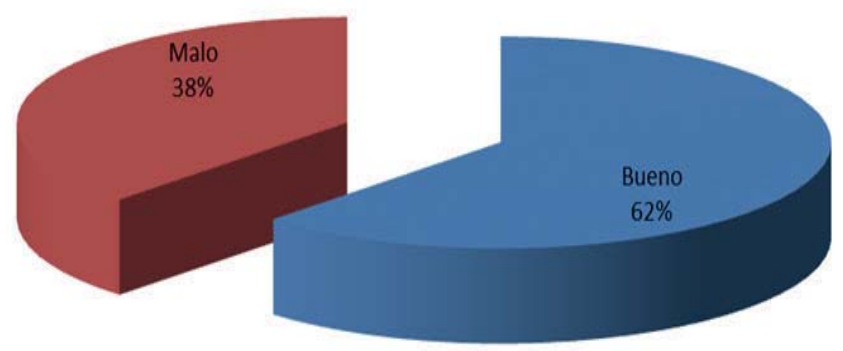

Figura 1. Resultado final del porcentaje de mejoría en los pacientes con enfermedad de Parkinson
Para analizar diferencias de los puntajes de mejoría UPDRS, antes y después de la intervención con células madre, se compararon promedios de puntaje. Observamos que, el puntaje medio y mediano, UPDRS, antes de la intervención fue 73.48 y 65 respectivamente. Luego de la intervención el puntaje medio y mediano UPDRS fue 30.09 y 22 respectivamente, como se puede observar en la tabla 4. Estas diferencias observados fueron estadísticamente significativas $(p<0.05)$ el mayor porcentaje de mejoría hallado fue de $98.6 \%$ y el menor fue de $5.13 \%$.

Tabla 4. Comparación de los resultados UPDRS en los pacientes con enfermedad de Parkinson

\begin{tabular}{|c|c|c|c|c|c|}
\hline Puntaje & Media & DS & Mediana & z & $\mathbf{P}$ \\
\hline UPDRS Antes & 73.48 & 37.81 & 65 & & 0.00 \\
\hline $\begin{array}{l}\text { UPDRS mejor valor } \\
\text { de Seguimiento }\end{array}$ & 30.09 & 23.95 & 22 & -6.62 & 0 \\
\hline
\end{tabular}

Aplicando T de Wilcoxon

En la figura 2, se aprecia una diferencia mayor entre los cuartiles uno, dos y tres, comparando los valores del score UPDRS antes y después de la intervención, con una mayor diferencia en los cuartiles superiores. Además, el $50 \%$ de pacientes del medio del grupo, también muestra gran cambio, antes y después de la intervención. En ambos casos, la mayor cantidad de pacientes se encuentran con una pequeña desviación derecha, es decir, tienen menor afección, según la medida de UPDRS.

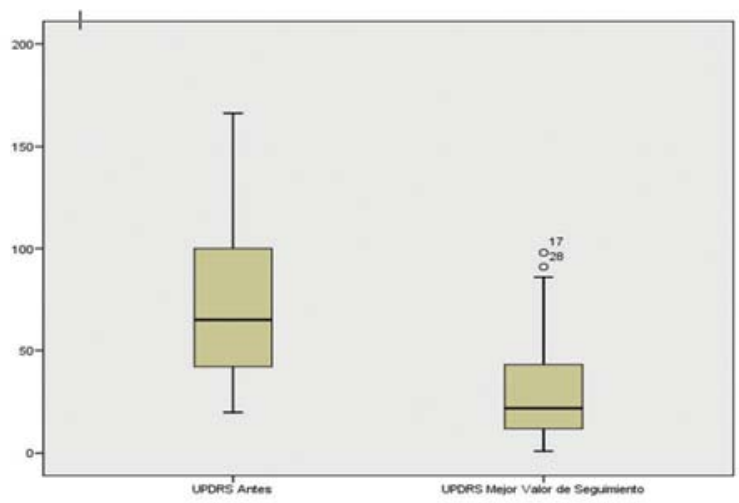

Figura 2. Diagrama de Cajas y Bigotes comparando resultados UPDRS en los pacientes con enfermedad de Parkinson 
No se encontraron diferencias significativas $(p>0.05)$ en los resultados de mejoría según sexo, ver tabla 5 , ya que el mayor porcentaj e de los pacientes que tuvieron resultado de mej oría bueno fueron varones (77.78\%), y los que tuvieron resultado de mejoría malo el mayor porcentaje fueron varones (54.55\%).

Tabla 5. Relación entre sexo y resultado de mejoría

\begin{tabular}{|lccc}
\hline Sexo & Bueno & Malo & Total \\
\hline Femenino & $8(22.22)$ & $10(45.45)$ & $18(31.03)$ \\
\hline Masculino & $28(77.78)$ & $12(54.55)$ & $40(68.97)$ \\
\hline Total & $36(100.0)$ & $22(100.0)$ & $58(100.0)$ \\
\hline$x^{2}=3.44$ & & & $p=0.06$ \\
\hline
\end{tabular}

Observamos diferencias significativas $(p<0.05)$, en los resultados de mejoría según grupos etáreos, ya que el mayor porcentaje de los pacientes que tuvieron un buen resultado, fueron aquellos que tenían edades entre 50 a 59 años (41.67\%), y los que tuvieron mal resultado, fueron pacientes con edades de 70 a 79 años (40.91\%), ver en tabla 6 .

Tabla 6. Relación entre edad y resultado de mejoría

\begin{tabular}{crrr}
\hline Edades & $\begin{array}{c}\text { Bueno } \\
\mathrm{n}(\%)\end{array}$ & $\begin{array}{c}\text { Malo } \\
\mathrm{n}(\%)\end{array}$ & \multicolumn{1}{c}{ Total } \\
\hline $30-39$ & $2(5.56)$ & $1(4.55)$ & $3(5.17)$ \\
\hline $40-49$ & $3(8.33)$ & $1(4.55)$ & $4(6.89)$ \\
\hline $50-59$ & $15(41.67)$ & $2(9.09)$ & $17(29.31)$ \\
\hline $60-69$ & $13(36.11)$ & $8(36.36)$ & $21(36.21)$ \\
\hline $70-79$ & $3(8.33)$ & $9(40.91)$ & $12(20.68)$ \\
\hline $80-89$ & $0(0.0)$ & $1(4.55)$ & $1(1.72)$ \\
\hline Total & $36(100.00)$ & $22(100.00)$ & $58(100.00)$ \\
\hline$x^{2}$ & & & $p=0.02$
\end{tabular}

En la tabla 7 apreciamos que la presencia de factores intrínsecos, como diskinesia grave, hipertensión arterial, diabetes mellitus, cáncer en remisión y antecedentes familiares de EP, fueron muy bajos en los pacientes con enfermedad de Parkinson. Se observó que ninguno de los factores mencionados influyeron en el resultado de mejoría ( $p>0.05)$.

Tabla 7. Relación entre factores intrínsecos y resultado de mejoría

\begin{tabular}{lccc}
\hline \multicolumn{1}{|c}{ Variable } & \multicolumn{3}{c}{ Resultado de mejoría } \\
\hline Bueno & $\begin{array}{c}\text { Malo } \\
\mathrm{n}(\%)\end{array}$ & $\mathbf{p}$ \\
\hline Diskinesia grave & $3(8.3)$ & $1(4.5)$ & 0.58 \\
Hipertensión arterial & $0(0.0)$ & $1(4.5)$ & 0.20 \\
Diabetes mellitus & $5(13.9)$ & $1(4.5)$ & 0.26 \\
Cáncer & $1(2.8)$ & $0(0.0)$ & 0.43 \\
Antecedente familiar & $3(8.3)$ & $0(0.0)$ & 0.16
\end{tabular}

En la tabla 8 apreciamos que la edad al inicio de la enfermedad, difiere según resultado de mejoría, encontrando que, en edades tempranas, se han tenido mejores resultados de mejoría $(p<0.05)$. Las otras variables; mes de seguimiento en su mejor mes y tiempo de enfermedad al implante, no han influido en el resultado de mejoría ( $p>0.05)$.

Tabla 8. Comparación de mes de seguimiento, edad inicio de enfermedad y tiempo de enfermedad, según resultado de mejoría

\begin{tabular}{lllrrr}
\multicolumn{1}{c}{ Variables } & Mejoría & $\mathbf{n}$ & Media & DS & $\mathbf{p}$ \\
& Bueno & 36 & 4.42 & 3.53 & \\
$\begin{array}{l}\text { Mes de Seguimiento } \\
\text { en su mejor mes }\end{array}$ & Malo & 22 & 3.64 & 3.24 & 0.23 \\
& Bueno & 36 & 49.83 & 9.85 & \\
$\begin{array}{l}\text { Edad al inicio de } \\
\text { la enfermedad }\end{array}$ & Malo & 22 & 55.91 & 12.70 & 0.02 \\
$\begin{array}{l}\text { Tiempo de Enfermedad } \\
\text { al implante }\end{array}$ & Bueno & 36 & 8.50 & 4.34 & \\
& Malo & 22 & 10.55 & 6.32 & 0.34 \\
& & & & &
\end{tabular}

Aplicando U de

Mann Whitney 


\section{DISCUSIÓN}

La EP afecta hasta el $2 \%$ de la población mayor de 65 años, con una mayor incidencia en hombres que en mujeres, pero de similar distribución en todos los grupos étnicos (1-3).

En el presente estudio se confirman tales aseveraciones, ya que el mayor porcentaje de los pacientes fueron varones y no las mujeres; además, el mayor porcentaje de pacientes tenían edades mayores a 60 años.

La respuesta clínica a la terapia con células madre adultas autólogas se midió de acuerdo a una escala internacional reconocida, la UPDRS [rango: 0 (sin signos de discapacidad o síntomas) a 199 (completa discapacidad y síntomas severos)].

En este sentido, el porcentaje de respuesta clínica promedio y mediano, fue $59 \%$ y $60 \%$, respectivamente; dichos porcentajes, posteriormente, fueron agrupados en mejoría clínica buena (buen resultado: mayor a 50\%) y mejoría clínica mala (mal resultado: menor a 50\%). La mayoría de pacientes lograron un buen resultado con la terapia.

Es importante resaltar que, estudios realizados en ratas con Parkinson, también mostraron mejorías, luego de tratamientos con células madre, aunque usaron células embrionarias (17-20); otros estudios similares ofrecieron mejorías también, pero reportaron la aparición de tumores de tipo teratoma, en las regiones tratadas con células madre embrionarias (21-23).

En pacientes humanos con EP, también se han realizado estudios en los cuales administrando una proteína estimuladora de células madre neurales, se lograron mejorías en los síntomas (24-26).

Por otro lado, si comparamos estudios realizados con células madre adultas, podemos observar que Dezawa et al (27) encontró que células madre mesenquimales de médula ósea diferenciadas, también lograron mejoría en ratas con EP. Murell W et al (28) encontró que células madre de las fosas nasales diferenciadas también lograron mejoría en ratas con EP. Weiss ML et al (29) usó células madre de cordón umbilical para tratar ratas con Parkinson y logró mejoría de síntomas motores y de comportamiento, similar a lo hallado en este estudio.

En pacientes humanos con EP, Levesque MF et al (30) usa las células madre adultas autólogas, las mismas que fueron usadas en nuestro estudio de Brazzini et al (31), y evaluadas en este estudio.

Levesque MF et al además, cultiva y diferencia las células in vitro, y luego éstas son microinjectadas, demostrando que alivian los síntomas de un paciente con EP, durante al menos cinco años.

Este tiempo de seguimiento es mayor al nuestro, además encuentran una mejoría de hasta $83 \%$ en la UPDRS, mayor que la media de los pacientes evaluados en este estudio, pero no mayor que al encontrado en nuestro paciente con mayor mejoría.

En nuestra investigación no se encontró una relación significativa entre tiempo de enfermedad y mejoría con la terapia celular, empleando la prueba de Chi-cuadrado ( $p>0.05)$, demostrando que ambas variables son independientes.

Es interesante que, analizando la relación entre edad y sexo con mejoría del tratamiento con la prueba de Chi-cuadrado, se haya encontrado que la edad de los pacientes influyen $(p<0.05)$ en la mejoría de los pacientes con EP.

Entre los que tuvieron buenos resultados de mejoría, la mayoría de pacientes tenían entre 50 a 59 años (41.67\%), y entre los que tuvieron mal resultado, la mayoría de pacientes tenían de 70 a 79 años (40.91\%).

También debemos destacar que la edad al inicio de la enfermedad, influyó en el resultado de mejoría, encontrando que en edades tempranas se ha tenido mejores resultados de mejoría $(p<0.05)$.

Por último, sobre la presencia de factores intrínsecos como diskinesia grave, hipertensión 
arterial, diabetes mellitus, cáncer y antecedentes familiares de EP, fueron muy baj os en los pacientes con enfermedad de Parkinson. Por lo tanto, se observó que ninguno de los factores mencionados influyeron en el resultado de mejoría ( $p>0.05)$. Sin embargo, es posible que la baja incidencia en algunos de estos factores, haya condicionado esta falta de relación.

\section{CONCLUSIONES}

Los factores que influyen en la mejoría de los pacientes tratados con terapia celular para EP son la edad de los pacientes al inicio de la enfermedad y la edad al tratamiento. Por lo que se recomienda que los métodos para elegir a los pacientes que recibirán esta terapia, incluyan estos factores. Además, recomendamos realizar un estudio con mayor cantidad de pacientes para poder evaluar mejor las relaciones en los factores donde la prevalencia fue muy baja. También es posible que si un paciente que inició su enfermedad más joven, también haya recibido terapia celular más joven. Por lo tanto, sería importante ver si existe una relación de dependencia entre edad al inicio de enfermedad y edad al tratamiento con células madre.

\section{Agradecimiento}

Dr. Carlos León, Dr. Augusto Brazzini, Dr. Raúl Cantella, Sra. Ana Rosa Brazzini, Dra. Mariana Brazzini, Mg. Jorge Medina.

\section{Fuentes de financiamiento}

Este artículo ha sido financiado por el autor.

\section{Conflictos de interés}

El autor declara no tener ningún conflicto de interés.

\section{REFERENCIAS BIBLIOGRÁFICAS}

1. Factor SA, Weiner WJ. Parkinson's Disease: Diagnosis and Clinical Management, 2nd Edition. 2 edition. New York: Demos Medical Publishing; 2007. 1000 p.

2. Nutt JG, Wooten GF. Clinical practice. Diagnosis and initial management of Parkinson's disease. N Engl J Med. 2005 Sep 8;353(10):1021-7.

3. Fischbach GD, McKhann GM. Cell therapy for Parkinson's disease. N Engl J Med. 2001 Mar 8;344(10):763-5.

4. Fernandez-Espejo E, Armengol JA, Flores JA, Galan-Rodriguez $B$, Ramiro S. Cells of the sympathoadrenal lineage: biological properties as donor tissue for cell replacement therapies for Parkinson's disease. Brain Res Rev. 2005;49:343-54.

5. Kordower JH, Freeman TB, Snow BJ, Vingerhoets FJ, Mufson EJ, Sanberg PR, et al. Neuropathological evidence of graft survival and striatal reinnervation after the transplantation of fetal mesencephalic tissue in a patient with Parkinson's disease. N Engl J Med. 1995 Apr 27;332(17):1118-24.

6. Piccini P, Brooks DJ, Björklund A, Gunn RN, Grasby PM, Rimoldi O, et al. Dopamine release from nigral transplants visualized in vivo in a Parkinson's patient. Nat Neurosci. 1999 Dec;2(12):1137-40.

7. Guttman M, Kish SJ, Furukawa Y. Current concepts in the diagnosis and management of Parkinson's disease. CMAJ Can Med Assoc J J Assoc Medicale Can. 2003 Feb 4;168(3):293-301.

8. Weissman IL. Stem cells: units of development, units of regeneration, and units in evolution. Cell. 2000 Jan 7;100(1):15768.

9. Longo DL, editor. Harrison's principles of internal medicine. 18th ed. New York: McGraw-Hill; 2012. 426 p.

10. Lakshmipathy U, Hart RP. Concise review: MicroRNA expression in multipotent mesenchymal stromal cells. Stem Cells Dayt Ohio. 2008 Feb;26(2):356-63.

11. Krause DS. Plasticity of marrow-derived stem cells. Gene Ther. 2002 Jun;9(11):754-8.

12. He Q, Wan C, Li G. Concise review: multipotent mesenchymal stromal cells in blood. Stem Cells Dayt Ohio. 2007 Jan;25(1):6977. 
13. Sordi V, Malosio ML, Marchesi F, Mercalli A, Melzi R, Giordano T, et al. Bone marrow mesenchymal stem cells express a restricted set of functionally active chemokine receptors capable of promoting migration to pancreatic islets. Blood. 2005 Jul 15;106(2):419-27.

14. Gage FH, Fisher LJ. Intracerebral grafting: a tool for the neurobiologist. Neuron. $1991 \mathrm{Jan} ; 6(1): 1-12$.

15. Meyer GP, Wollert KC, Lotz J, Steffens J, Lippolt P, Fichtner S, et al. Intracoronary bone marrow cell transfer after myocardial infarction: eighteen months' follow-up data from the randomized, controlled BOOST (BOne marrOw transfer to enhance ST-elevation infarct regeneration) trial. Circulation. 2006 Mar 14;113(10):128794.

16. Assmus B, Schächinger V, Teupe C, Britten M, Lehmann R, Döbert $\mathrm{N}$, et al. Transplantation of Progenitor Cells and Regeneration Enhancement in Acute Myocardial Infarction (TOPCARE-AMI). Circulation. 2002 Dec 10;106(24):3009-17.

17. Chiba S, Lee YM, Zhou W, Freed CR. Noggin enhances dopamine neuron production from human embryonic stem cells and improves behavioral outcome after transplantation into Parkinsonian rats. Stem Cells Dayt Ohio. 2008 Nov;26(11):2810-20.

18. Takagi Y, Takahashi J, Saiki H, Morizane A, Hayashi T, Kishi Y, et al. Dopaminergic neurons generated from monkey embryonic stem cells function in a Parkinson primate model. J Clin Invest. 2005 Jan;115(1):102-9.

19. Ben-Hur T, Idelson M, Khaner H, Pera M, Reinhartz E, Itzik A, et al. Transplantation of human embryonic stem cell-derived neural progenitors improves behavioral deficit in Parkinsonian rats. Stem Cells Dayt Ohio. 2004;22(7):1246-55.

20. Kim J-H, Auerbach JM, Rodríguez-Gómez JA, Velasco I, Gavin D, Lumelsky $\mathrm{N}$, et al. Dopamine neurons derived from embryonic stem cells function in an animal model of Parkinson's disease. Nature. 2002 Jul 4;418(6893):50-6.

21. Roy NS, Cleren C, Singh SK, Yang L, Beal MF, Goldman SA. Functional engraftment of human ES cell-derived dopaminergic neurons enriched by coculture with telomerase-immortalized midbrain astrocytes. Nat Med. 2006 Nov;12(11):1259-68.

22. Nishimura F, Yoshikawa M, Kanda S, Nonaka M, Yokota H, Shiroi A, et al. Potential use of embryonic stem cells for the treatment of mouse parkinsonian models: improved behavior by transplantation of in vitro differentiated dopaminergic neurons from embryonic stem cells. Stem Cells Dayt Ohio. 2003;21(2):171-80.

23. Bjorklund LM, Sánchez-Pernaute R, Chung S, Andersson T, Chen IYC, MCNaught KSP, et al. Embryonic stem cells develop into functional dopaminergic neurons after transplantation in a Parkinson rat model. Proc Natl Acad Sci U S A. 2002 Feb 19;99(4):2344-9.

24. Love S, Plaha P, Patel NK, Hotton GR, Brooks DJ, Gill SS. Glial cell line-derived neurotrophic factor induces neuronal sprouting in human brain. Nat Med. $2005 \mathrm{Jul} ; 11(7): 703-4$.

25. Slevin JT, Gerhardt GA, Smith CD, Gash DM, Kryscio R, Young B. Improvement of bilateral motor functions in patients with Parkinson disease through the unilateral intraputaminal infusion of glial cell line-derived neurotrophic factor. J Neurosurg. 2005 Feb;102(2):216-22.

26. Gill SS, Patel NK, Hotton GR, O'Sullivan K, McCarter R, Bunnage M, et al. Direct brain infusion of glial cell line-derived neurotrophic factor in Parkinson disease. Nat Med. 2003 May;9(5):589-95.
27. Dezawa M, Kanno H, Hoshino M, Cho H, Matsumoto N, Itokazu Y, et al. Specific induction of neuronal cells from bone marrow stromal cells and application for autologous transplantation. J Clin Invest. 2004 Jun;113(12):1701-10.

28. Murrell W, Wetzig A, Donnellan M, Féron F, Burne T, Meedeniya A, et al. Olfactory mucosa is a potential source for autologous stem cell therapy for Parkinson's disease. Stem Cells Dayt Ohio. 2008 Aug;26(8):2183-92.

29. Weiss ML, Medicetty S, Bledsoe AR, Rachakatla RS, Choi M, Merchav $\mathrm{S}$, et al. Human umbilical cord matrix stem cells: preliminary characterization and effect of transplantation in a rodent model of Parkinson's disease. Stem Cells Dayt Ohio. 2006 Mar;24(3):78192.

30. Levesque MF, Neuman T, Rezak M. Therapeutic Microinjection of Autologous Adult Human Neural Stem Cells and Differentiated Neurons for Parkinson's Disease: Five-Year Post-Operative Outcome. Open Stem Cell J. 2009;1:20-9.

31. Brazzini A, Cantella R, De la Cruz A, Yupanqui J, León C, Jorquiera T, et al. Intraarterial autologous implantation of adult stem cells for patients with Parkinson disease. J Vasc Interv Radiol JVIR. 2010 Apr;21(4):443-51

\section{Correspondencia:}

Tamara Jorquiera - Johnson

Dirección: Av. El corregidor 1531, La Molina, Lima-Perú

Teléfono: $365-2300$

Correo electrónico: tjorquieraj@usmp.pe 